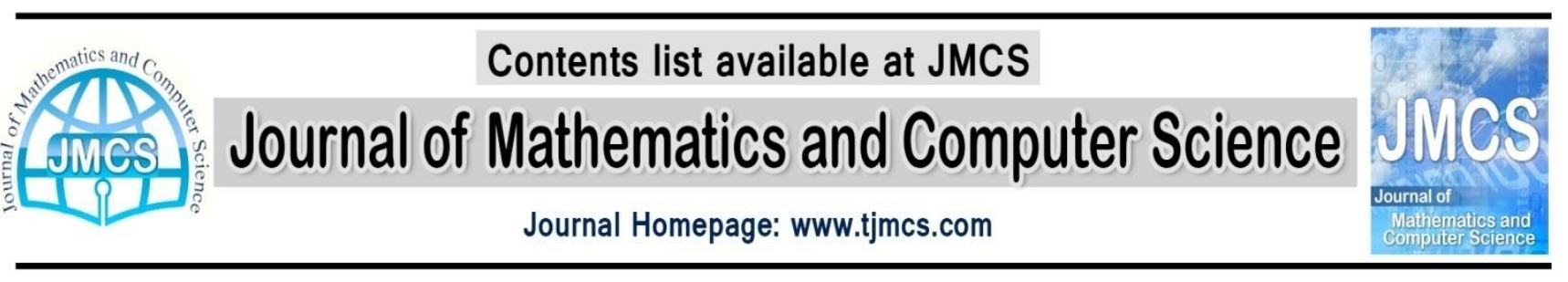

\title{
ETDWSN: An method for energy efficiency increase by combining the index parameters in wireless sensor networks
}

\author{
Fatollah Rouhi ${ }^{1}$, Arash Ghorbannia Delavar ${ }^{2}$, Sina Hedayati ${ }^{3}$ \\ Department of Computer,Payame Noor University,PO BOX 19395-3697, Tehran, IRAN
}

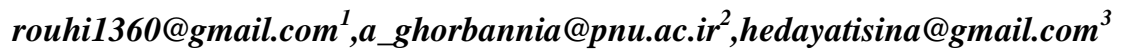

Article history:

Received May 2014

Accepted June 2014

Available online June 2014

\begin{abstract}
The development of routing algorithms in wireless sensor networks, energy supply increase in the dependent nodes is by viewing the previous algorithms on the data-centric routing algorithm, the method suggested in ETDWSN we offer combined with the index parameters of the method, we've created suitability function in the ETDWSN method we've reduced energy consumption and also in this case the sensor system network efficiency is increased in The correct using proposals could increase the energy threshold detector on wireless sensor network we have in ETDWSN method with the index parameters select the weight goal we've provided compared with neighboring nodes selection action relative to the source node, we improve the routing space finally The average delay time of the nodes to the Auto GBR ·GBR ، GBR-C and have lowered.
\end{abstract}

Keywords: Efficiency‘FunctionWeigh، Wireless Sensor Network, Algorithm ETDWSN, Distributed Node.

\section{Introduction}

Since the wireless sensor network 2000 as a scientific event is located, Wireless Sensor Network (WSN) consists of many nodes which are randomly developed based on the specific circumstances. Wireless Sensor Networks are used as a technology to supply the sensitive geographical equipment in the large development area of these electronic devices [1]. The applications of Wireless Sensor Network includesecurity detection systems, surveillance [2], military, target tracking, habitat monitoring [3], 
medical industry and its applications [4] and likeso many articles have reviewed the energy efficiency in Wireless Sensor Networks. In a wireless sensor network, the request message is broadcasted to all nodes on the network by the base station. . Data are transferred to a sink for further processing by the network nodes (as sink nodes).the energy consumption depends on the processing time and data transmission time among them. so, our purpose in this article is to reduce retransmission of data in a network and to find the more optimal way to reduce the energy consumption in nodes. Since wireless sensor networks have limited access to energy, energy consumption is one of the most important items for wireless sensor networks.

Each node independently finds its next leap into the sink and highlights a direct path for transmission that saves high energy. This scheme is used for the sensor networks which are in a restricted area. This scheme, beside being able to broadcast and balance a network, is used to reduce transmissions and saving energy.Many articles focus on the data routing with energy constraints. Many to strategies proposed in the data broadcast and ability to balance network and reducing data transmissions, such Geographic Adaptive Fidelity (GAF) [5], Low Energy Adaptive Clustering Hierarchy (LEACH) [6], Threshold sensitive Energy Efficient Network protocol (TEEN) [7], and Gradient-Based Routing (GBR) [8], are among the routing proposed in literature. LEATH and TEEN are cluster-based routing protocols. The extra overheads of forming clusters diminish the gain in energy consumption. GAF and GBR are locationbased routing protocols. In GAF, the GPS information is needed by each node to set its location which consequently increases the cost of each node. GBR is developed from the Directed Diffusion protocol[9]. The basic idea record the number of hops when a nod receives a message; then, each node sets up its height according to the minimum number of hops. The heights' difference between a node and its neighbours forms the gradient on the corresponding link, many articles have developed GBR protocol using auxiliary techniques for data aggregation and data traffic

\section{2. RELATED WORKS}

This paper aims to show modify GBR protocol in energy consumption. Since the proposed approach in this article is to solve problems a GBR protocol using Network coding method, [10] several paths are combined with routing in network coding. The sink receives its message from the source. Before the node forwards source message, it must be aware of the rout regardless them. The advantage of this algorithm is that it is reliable and guarantees the energy saving. The main idea of competitive algorithm is exploiting the wireless environment, which includes aa routes rather than a traditional path and includes a message driving that selects and sends the message properly. In the schemes based on CBF, the main task of source node is to distribute data packets to all neighbors and then forwards data by one of its best nodes [11]. To do this, three suppression plans are presented: public suppression, regional suppression and transmission suppression. The aim of suppression algorithm is to prevent many nodes and duplicate packets.

These three mentioned schemes are to prevent duplicate packets but under the specific circumstances. Novel Message Forwarding Technique on the basis including geographic position is randomly selected through the connections among them. A receiver which is closer to the destination has high priority. As 
a result, the nodes closed to the destination are constantly selected and in the effect of the same energy, these nodes arrive to the end and disappear sooner. Integration routing and MAC protocol enhance throughput for transmission over a wireless and multi-hop network.[12] The performance of EXOR in a group selects the source nodes which have a list of driving positions based on the priority of closeness to the destination. The algorithm which loads the intended progress based on the priority of message forwarding is EPA that is a rate in order to obtain maximum power.

LNCS is a protocol for sensor network that is able to divide the data about location used to several nonoverlapping cells for data availability. [13] SBLNC is a protocol which guarantees the security of data broadcast using linear coding network in wireless sensor networks. In [14], the authors propose an adaptive network coding approach to enhance reliability in WSNs by considering redundancy. An analytic model is then proposed to estimate the proper a redundancy. Further, they propose a clusterbased and distributed scheme to dynamically adjust the redundancy at each hop. but, this algorithm considers redundancy to guarantee the reliability which is not suitable for the broadcast scenario.

Network coding is forwarding data based on the slope of connections among them. Data are always sending on the shortest route. GBR is able to select the shortest route toward the sink and balance the energy in the whole rout.

Protocols GBR and GBR-NC is provide on Network Coding and the average (including received and sent) traffic for each node in GBR and GBR-NC can be determined as:[3]

$$
\begin{aligned}
& \mathrm{T}_{\mathrm{GBR}}=\mathrm{M} * \mathrm{~S}_{\text {interest }} *\left(1+\mathrm{N}_{\mathrm{AVG} . \text { neighøur }}\right) \\
& \mathrm{T}_{\mathrm{GBR}-\mathrm{NC}}=\left(\mathrm{ANP}_{\mathrm{GBR}-\mathrm{NC}} *\left(\mathrm{~S}_{\text {interest }}+\mathrm{N} * \mathrm{~S}_{\text {Cofficient }}\right)+\frac{\mathrm{NP}_{\mathrm{NACK}} * \mathrm{~S}_{\mathrm{NACK}}}{\mathrm{N}_{\mathrm{NODE}}}\right) *\left(1+\mathrm{N}_{\mathrm{AVG} . \text { neighbur }}\right) \\
& \mathrm{T}_{\mathrm{TDWSN}}=\mathrm{ANP}_{\text {n.neighbou }}+\mathrm{NP}_{\text {n.candidae }} *\left(1+\mathrm{NP}_{\text {s.node }}\right)
\end{aligned}
$$

Energy consumption for each node in GBR and can be determined as:[3]

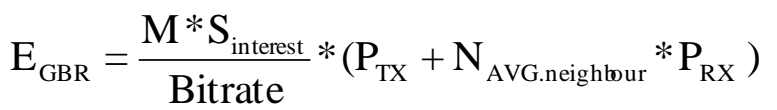

Energy consumption for each node in GBR-NC and can be determined as:[3]

(5)

$$
\mathrm{E}_{\mathrm{GBR}-\mathrm{NC}}=\frac{1}{\text { Bitrate }} *\left(\mathrm{ANP}_{\mathrm{GBR}-\mathrm{NC}} *\left(\mathrm{~S}_{\text {interest }}+\mathrm{N} * \mathrm{~S}_{\text {confficient }}\right)+\frac{\mathrm{NP}_{\mathrm{NACK}} * \mathrm{~S}_{\mathrm{NACK}}}{N_{\text {Node }}} *\left(\mathrm{P}_{\mathrm{TX}}+\mathrm{N}_{\text {AVG.neighbur }} * \mathrm{P}_{\mathrm{RX}}\right)\right.
$$

[3]Energy consumption competitive approach 


$$
\begin{aligned}
& \mathrm{E}=\left(\mathrm{P}_{\mathrm{TX}}+\mathrm{P}_{\mathrm{RX}}\right) \Delta \mathrm{T} \\
& \Delta \mathrm{T}=\frac{\mathrm{S}_{\text {Data }}}{\mathrm{P}} / \text { Bitrate }
\end{aligned}
$$

[3]Energy consumption for a node in competitive approach

$$
\mathrm{E}=\frac{\mathrm{P}_{\mathrm{TX}}+\mathrm{P}_{\mathrm{RX}}}{\text { Bitrate }} * \frac{\mathrm{S}_{\text {Data }}}{\mathrm{P}}
$$

$$
\mathrm{E}=\frac{\mathrm{P}_{\mathrm{TX}}+\mathrm{n} * \mathrm{P}_{\mathrm{RX}}}{\text { Bitrate }} * \frac{\mathrm{S}_{\text {Data }}}{1-(1-\mathrm{P})^{\mathrm{n}}} \ldots \ldots \ldots \ldots \ldots \ldots . . . \cdots=1,2,3,4,5
$$

The first problem in the GBR-NC competitive algorithm of the method selecting receiver node of neighbors in the network coding and secondly,this algorithm is only dependent on a parameter.

ETDWSN approach are proposed to solve the above problems with consider special parameters and threshold for selectingbest candidate node. This approach procesing time and consumption energy is optimized.

\section{PROPOSED ALGORITHM}

Competitive selection approach have corrected nodes in GBR for saving more energy in the nodes and Increase the flexibility of the proposed algorithm. Then, the energy consumption for GBR is analyzed to determine the number of candidate forwards.

The aim of this paper is Competitive approach to select the nodes to be removed. Selection of candidate nodes from the source node to neighboring nodes by the combination of special parameters is to reduce the retransmission in order to save the energy of the wireless sensor network.

the Source node selected the candidate node from neighbor nodes. The approach selection for candidate node is dependent on special parameters. selected candidate node by send packet distance to neighbors. neighbor nodes get packet distance if the distance is less than the threshold are set selected to candidate node. This approach isreduce retransmission interest message to more save the energy of node in sensor network.

The candidate node to send ACK to source node and the source node received the ACK. which source node how many ACK candidate node is received.the ACK number equal to the number of candidate node.According to number of ACK determined how to send data

If number of ACK equal to one, send the data to candidate node and forwarding data to sink. 
If the number of ACK equal to two or more, the source node can prioritize candidate nodes and source node decides which candidates node to send. Since receiving packets also consume energy in the proposed algorithm source node does not broadcasting packets to all neighbors. As a result, with load balancing, and the reduction in the number of transmissions in order to save energy wireless sensor network.

Source node when for selecting candidate node to have more than one condition.time and returned to the source node and the candidate node computes.

If the total time for sending and receiving packets is less than the threshold the selected nodes as candidate nodes While there is not the two node similar to in the time and weight parameterelse if send to message randomly One of the nodes

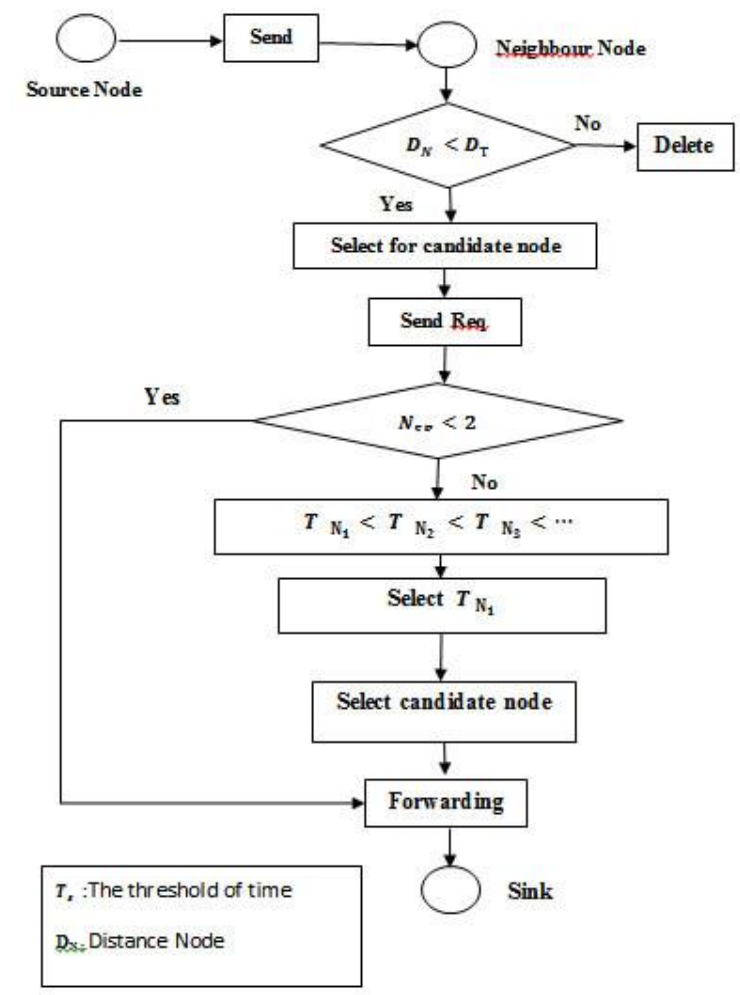

\subsection{RELIABILITY ALGORITHM}

The main aim of the proposed in the approach supposed that our messages reach to the destination intact. In sensor network it is important that ensure the data arrives intact to the destination in every hop. Our presented algorithm is based on GBR protocol that investigates the state of the nodes hoping in every leap. The performance is presented in figure 2 .

1- The source node broadcasts to the interest message all neighbor node. 
2- All nodes save the number of remaining hops to the sink in their lists. When the nodes receive the messages, Neighbors are in the request message list,less than a threshold distance from the source node for set as candidate node.

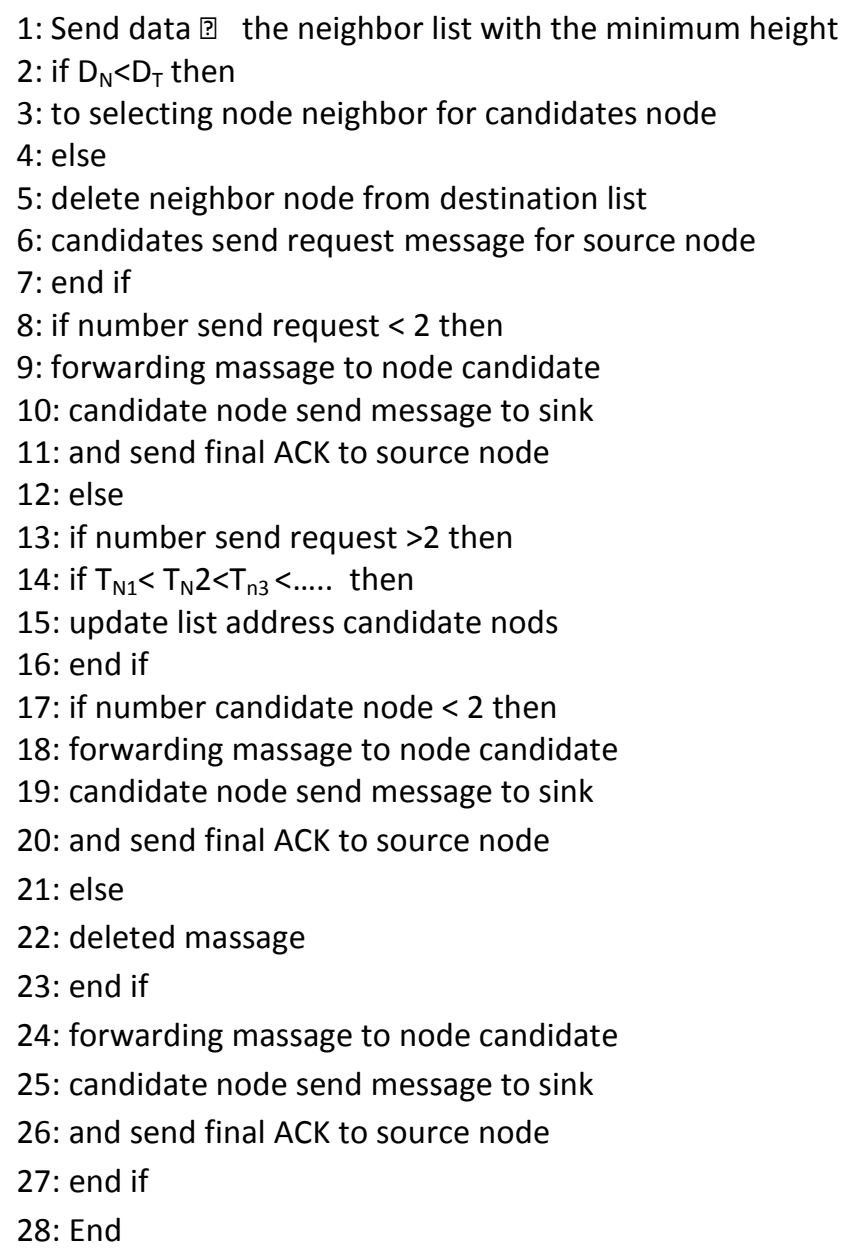

3- The receiver of message receives the number of forward positions from destination address table.

4- The candidate node send to request massage to source node.

5- If number of ACK equal to one, send the data to candidate node and forwarding data to sink, and send to source node final ACK

6- If the number of ACK equal to two or more, the source node can prioritize candidate nodes and source node decides which candidates node to send.

7- Time and returned to the source node and the candidate node computes. selected candidate nodeif time send and return less is threshold time and update destination list 
8- Otherwise, we consider the weight parameter and there is a counter for the number of the packets of transmitted per node.the node is selected if the counter showed number of packet is fewer.

9- The counter with a lower value equals is the node with more weight.As a result, it is Priority to select the source node

Finally the selected candidate node sending packet to sink and sending final ACK to source node.

One important advantage of this algorithm is load balancing is consumption energy in sensor network.

\subsection{ANALYSIS OF ENERGY CONSUMPTION}

In the approach ETDWSN, Energy consumption is calculated using the specific parameters in sensor networks. We were able to more energy efficiency in sensor networks, send and receive packets to the source node analysis.

Based on the number of candidate nodes that are within the threshold, the source node selects an optimal path. According to the calculations hops distance is too difficult work. But we were able to use certain parameters and consider the distance threshold to predict and estimate. This paper has shown that we can calculate the distance to the bandwidth and time send data and receive data independent on source node.

Important in estimating distances, time delays in the candidate nodes. Delay for a node is calculated as follows:

$\mathrm{D}_{\text {elay }}=\left(\mathrm{N}_{\text {Send }} * \mathrm{~T}_{\text {Wait }}\right)-\left(\mathrm{T}_{\mathrm{ACK}}+\mathrm{T}_{\mathrm{REQ}}\right)$

Obtain delay can hop distance between nodes can be calculated:

$\mathrm{D}_{\text {ist }}=\mathrm{B}_{\mathrm{W}} *\left(\Delta T-D_{\text {elay }}\right)$

The result is shown for a candidate nodes:

$\mathrm{E}_{\mathrm{f}}=\frac{\Delta T-D_{\text {elay }}}{D_{\text {ist }}} * \frac{S_{\text {Data }}}{B W}$ 
If the number ofcandidate nodesismore than oneforagiven:

If $\mathrm{n}>1$ then: $\quad \mathrm{D}_{\text {elay }}=\left(\sum_{n=h o p}^{1} N_{\text {Send }} * \sum_{T=h o p}^{1} T_{\text {Wait }}\right)-\left(T_{A C K}+\left(T_{R E Q} * n\right)\right)$

$\mathrm{D}_{\text {ist }}=B_{B W} *\left(\sum_{n=h o p}^{1} \Delta T_{n}-\sum_{n=\text { Send }} D_{\text {elay }}\right)$

In the ETDWSN's approach has tried, the special parameters would take Threshold to select candidate nodes.

$\mathrm{E}_{\mathrm{f}}=\frac{\sum_{n=h o p} \Delta T_{n}-\sum_{n=\text { Send }} D_{\text {elay }}}{\sum_{n=h o p} D_{\text {ist }}} * \frac{S_{\text {Data }}}{B W}$

The proposed algorithm increases the candidate nodes to send data, across the networkenergy consumption will be balanced.

Energy consumption range indicates that with comparison minimize and optimal mode, bycontrol candidate nodes in sensor networks is reducedhe consumption energy intervals is decreased and in the approach ETDWSN very important because with increase neighbor nodes consumption energy a Little change and while balancing the energy of the whole network.

\section{SIMULATIONS AND RESULT}

Considering the number of wireless sensor network nodes that are randomly scattered in the environment,simulationis done.in ETDWSN the network coding scheme is set according to the network density. Each node in the networks has fixed radio rang of $180 \mathrm{~m}$.the energy framework 2.0 provided in the OMNET++ is used and each node is assigned with the same initial energy capacity of $30 \mathrm{j}$ at beginning of the simulation

Mica2 power consumption model presented in[15] is used in which the radio consumes21.5 $\mathrm{mA}$ at $+10 \mathrm{dbm}$ for transmitting and $7 \mathrm{mAfor}$ receiving under a $3 \mathrm{v}$ power supply.

In fegure3 : consumption energy for random sensor network is showed. simulation result show that proposed apporach give better than result when compare to the last algorithm 


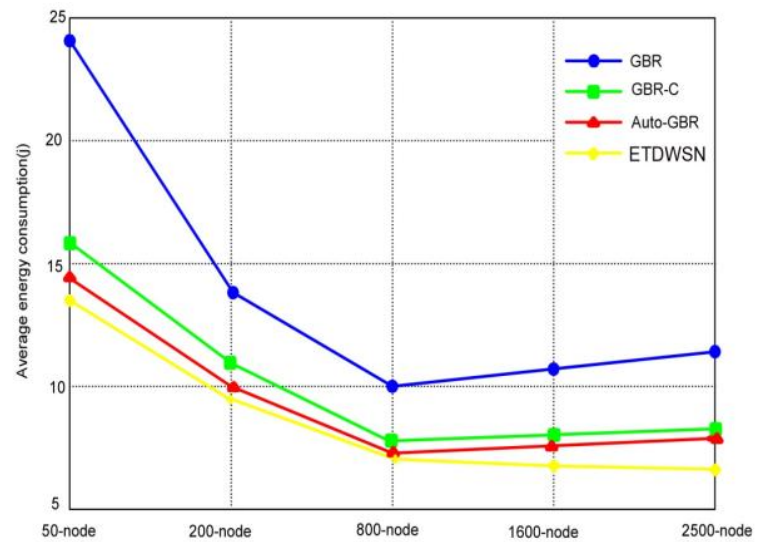

Figure 3:Average energy consumption for different networks

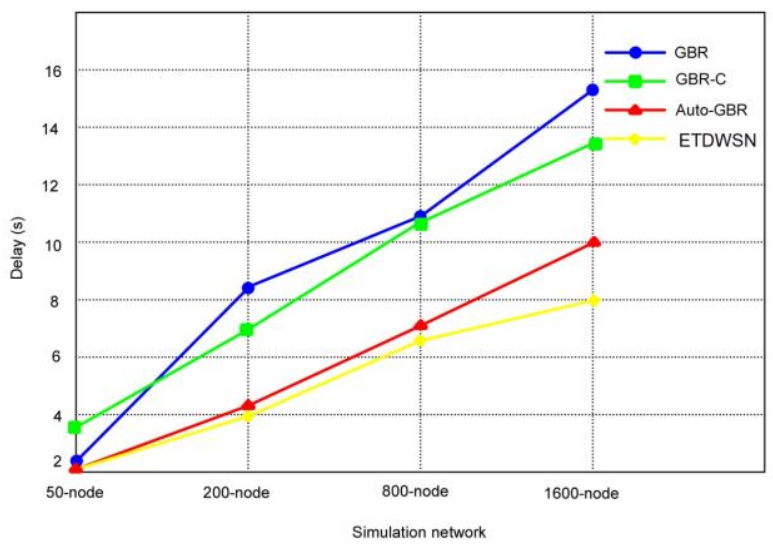

Figure4: The transmission delay for random networks

Figure 4 shows The number of request packages for each node in the second in wireless sensor network node.it can also be seen that there are some node Data transmission time is reduced.

Neighbors node toRestrictthe selection of candidates node in ordertoavoidduplicationofdata transmissionbecomes Itisrelated tosavingenergy, which increases networklifetime.

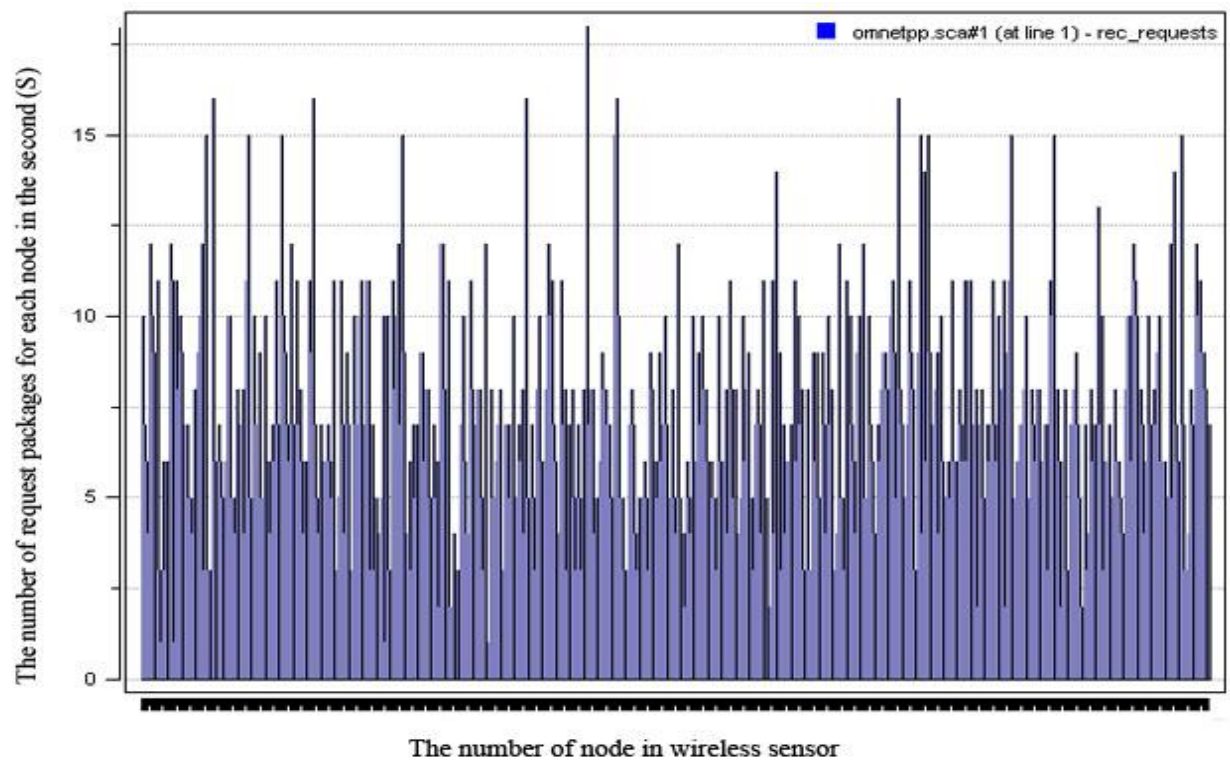

Figure 4 :The number of request packages for each node in the second 
Figure 5 shows The time of receive data which were sent by each node in wireless sensornetwork500node it can also be seen that there are more node receive Datatransmission timeis reduced.
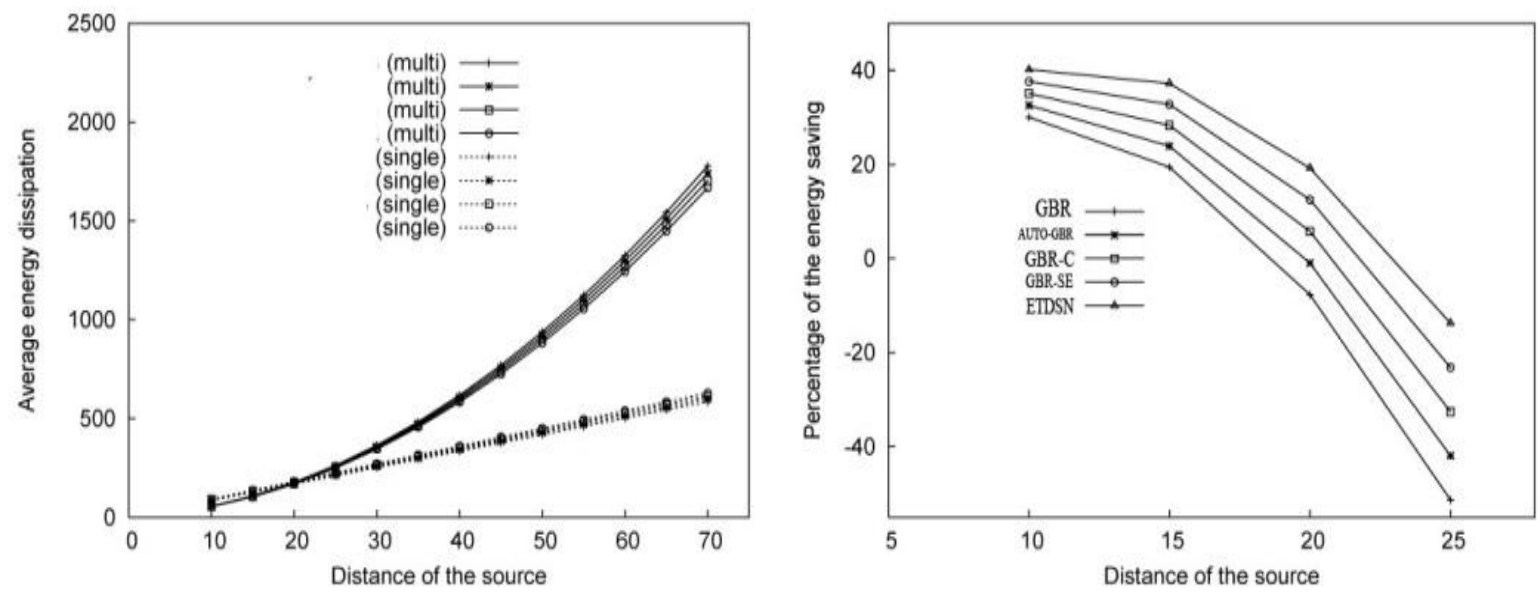

Figure 5:Time sleep of nodes for wireless sensor network

Charts provided by authors show the behavior of all nodes in a wireless sensor network. Each of which represents the behavior each nodes in the time of send data and time of receive data and time of sleep data.

According to the simulation results, we can see that this optimization algorithm more increase better than the previous algorithms have been presented in wireless sensor networks. The best selected for forwarding data and the most sleep time of node and the better selected for path for transmitting data

Increasing of node in the wireless sensor network on the optimization algorithm GBR-SE is not dramatically reduce the energy consumption. But thesealgorithms in wireless sensor networks always keeps the balance energy consumption.

\section{CONCLUSION}

The results of experiments show that GBR-SE algorithm is improved and it has a better efficiency energy compared with the two other algorithms, GBR and GBR-NC. Average improvement of GBR-SE as compared to GBR and GBR-C, Simulation results showed that compared with GBR around 7\% energy consumption and GBR-NC around 1.8\% energy consumption and GBR-SE around $2.1 \%$ energy consumption as well as network traffic is saved by ETDWSN. The next work can focus on probability distribution forresponding and concentrated processing and threshold distance. 


\section{REFERENCES}

1. SinaHedayati, ArashGhorbanniaDelavar, The method of GBR optimization by special parameters to decrease energy onsumption in WSNs Journal of mathematics and computer science 8 (2014), 387-397

2. ArashGhorbanniaDelavar, AbootorabAlirezaie, Amir Abbas Baradaran, KGAWSN: An Effective Way to Reduce Energy Consumption in Wireless Sensor Networks by Kmeans and Genetic Algorithms, International Journal of Computer Applications (0975 - 888) Volume 48- No.12, June 2012

3. V. Shnayder, M. Hempstead, B.-r. Chen, G.W. Allen, M. Welsh, Simulating the power consumption of large-scale sensor network applications, in: Proceedings of the 2nd International Conference on Embedded Networked Sensor Systems, SenSys '04, ACM, New York,NY, USA, 2004, pp. 188-200. oi:http://doi.acm.org/10.1145/1031495.1031518.

4. T. He, S. Krishnamurthy, J.A. Stankovic, T. Abdelzaher, L. Luo, R. Stoleru,T. Yan, L. Gu, Energyefficient surveillance system using wireless sensor networks, in: Mobisys, ACM Press, 2004, pp. 270-283.

5. G. Tolle, J. Polastre, R. Szewczyk, D. Culler, N. Turner, K. Tu, S. Burgess, T. Dawson, P. Buonadonna, D. Gay. Hong, A macroscope in the redwoods, in: Proceedings of the 3rd International Conference on Embedded Networked Sensor Systems, SenSys '05, ACM, New York,NY, USA, 2005, pp. 51-63. Doi : http ://doi.acm.org /10.1145 /1098918 .1098925.

6. R.K. Ganti, P. Jayachandran, T.F. Abdelzaher, J.A. Stankovic, Satire: asoftware architecture for smart attire, in: Proceedings of the 4th International Conference on Mobile Systems, ,mobiSys'06,ACM,NewYork,NY,USA,2006,pp.11023.doi:http://doi.acm.org/10.1145/1134680.1134 693

7. J.N. Al-Karaki, A.E. Kamal, Routing techniques in wireless sensornetworks: a survey, IEEE Wireless Commun. Mag. 11 (6) (2004) 6-28, doi:10.1109/MWC.2004.1368893

8. L. Shan-Shan, Z. Pei-Dong, L. Xiang-Ke, C. Wei-Fang, P. Shao-Liang,Energy efficient multipath routing using network coding in wirelesssensor networks, in: T. Kunz, S. Ravi (Eds.), Ad-Hoc, Mobile, andWireless Networks, vol. 4104, Springer, Berlin/Heidelberg, 2006, pp.114-127.

9. Y.S.Y. Yang, C. Zhong, J. Yang, Energy efficient reliable multi-pathrouting using network coding for sensornetwork,IJCSNSInt. J.Comput. Sci. Network Secur.8(12)(2008)114-127.

10. L. Shan-Shan, Z. Pei-Dong, L. Xiang-Ke, C. Wei-Fang, P. Shao-Liang, Energy efficient multipath routing using network coding in wireless sensor networks, in: T. Kunz, S. Ravi (Eds.), Ad-Hoc, Mobile, and Wireless Networks, vol. 4104, Springer,Berlin/Heidelberg,2006,pp.114-127.

11. Z. Xiong, W. Liu, J. Huang, W. Cheng, B. Cheng, Network coding approach for intra-cluster information exchange in sensor networks,in: Proc. VTC-2007 Fall Vehicular Technology Conf. 2007 IEEE66th,2007,pp.164168.doi:10.1109/VETECF.2007.49

12. E. Ayday, F. Delgosha, F. Fekri, Location-aware security services for wireless sensor networks using network coding, in: Proc. 26th IEEE Int. Conf. Computer Communications, INFOCOM 2007, IEEE, 2007, pp. 1226-1234. doi:10.1109/INFCOM.2007.146

13. Z. Zhu, Q. Tan, P. Zhu, Q. Zheng. Security broadcast based on linear network coding in WSN, in: Proceedings of International Computer Science and Software Engineering Conference, vol. 3, 2008, pp. 919-922.doi:10.1109/CSSE.2008.676.

14. T.-G. Li, C.-C. Hsu, C.-F. Chou, On reliable transmission by adaptive network coding in wireless sensor networks, in: ICC '09. IEEE International Conference on Communications, 2009, pp. 1-5. doi:10.1109/ICC.2009.5199247

15. Lusheng Miao, KarimDjouani, AnishKurien, Guillaume Noel Network coding and competitive approach for gradient based routing in wireless sensor networks Ad Hoc Networks, Volume 10, Issue 6, August 2012, Pages 990-1008 . 